\title{
Clustering dynamics of Lagrangian tracers in free-surface flows
}

\author{
Jörg Schumacher ${ }^{1,2}$ and Bruno Eckhardt ${ }^{2}$ \\ 1 Department of Mechanical Engineering, Yale University, New Haven, Connecticut 06520-8284, USA \\ ${ }^{2}$ Fachbereich Physik, Philipps-Universität Marburg, D-35032 Marburg, Germany
}

(November 20, 2018)

\begin{abstract}
We study the formation of clusters of passive Lagrangian tracers in a non-smooth turbulent flow in a flat free-slip surface as a model for particle dynamics on free surfaces. Single particle and pair dispersion show different behavior for short and large times: on short times particles cluster exponentially rapidly until patches of the size of the divergence correlation length are depleted; on larger times the pair dispersion is dominated by almost ballistic hopping between clusters. We also find that the distribution of particle density is close to algebraic and can trace this back to the exponential distribution of the divergence field of the surface flow.
\end{abstract}

PACS: 83.50.Lh, 47.27.Eq, 47.27.Qb

The Lagrangian evolution of passive tracers in turbulent flows has attracted considerable attention recently. Particle tracking techniques have been developed that allow for a detailed observation of their motion even in high-Reynolds number turbulence [1] 3]. The statistics of few particle clusters has been used to obtain information on the Lagrangian statistics of the flow field [4. Simplified models of passive scalars evolution, e.g. the Kraichnan model with its delta-correlated random velocity fields, have provided important insights into the origin of intermittency corrections to scaling laws [5]. The advection of particles that are not neutrally buoyant gives rise to clustering and this process has been suggested to be essential for the formation of rain [6]. Similar clustering phenomena should appear for bubbles or inertial particles in turbulent flows [7]. The problem we consider here is the advection of Lagrangian particles in a flat free surface above a turbulent volume flow. Previous approaches to the particle advection in such (compressible) flows include random maps [8] and Kraichnan models with prescribed smooth [9] and non-smooth [10] spatial variations. Realistic flows have some finite time correlations, but, as we will demonstrate here, they show further differences: the distribution of values for the divergence of the flow field is exponential (and not Gaussian [9]), the distribution of density is algebraic (and not lognormal [9]) and the two-particle dispersion shows an almost ballistic regime for large separations. Furthermore, this behavior is outside the range of Kraichnan type models since the ratio between divergence and velocity gradient fluctuations is such that the surface flow belongs to a marginal situation where the Kraichnan models predict neither clustering nor exponential separation of particles [0,11, 12].

The experimental realization, the dynamics and the properties of the flat free surface flows that we consider have been discussed in detail before [11, 12. What is needed here is the presence of a $r^{2 / 3}$-scaling in the inertial subrange due to connection to bulk turbulence in the volume below, i.e. a non-smooth flow with finite time correlations which goes beyond all previous analytical approaches. On the numerical side we integrate particle trajectories using a bicubic spline interpolation which was checked by comparison with analytical examples 13. and with a direct spectral evaluation of the velocity between the grid meshs 14. The pseudospectral simulations of the Navier-Stokes flow are based on grids with $256 \times 256 \times 65$ nodes, with a forcing that maintains a fixed energy injection rate $\epsilon$ [15]. A Taylor-Reynolds number $R_{\lambda}=u_{x, r m s}^{2} /\left[\nu\left(\partial_{x} u_{x}\right)_{r m s}\right]=145$ was achieved and the Kolmogorov length $\eta=\left(\nu^{3} / \epsilon\right)^{1 / 4}$ is 0.8 grid spacings. The Kolmogorov time is $\tau_{\eta}=(\nu / \epsilon)^{1 / 2}$.

A typical particle distribution that emerges from a uniform initial distribution is shown in Fig. 1 (upper panel). The particle dynamics shows two time regimes, a quick clustering into elongated structures, followed by a slower exchange of particles between structures. Superimposed on the particle distribution we show the surface flow that can be considered as a superposition of an irrotational and a gradient part by the Helmholtz decomposition theorem,

$$
\mathbf{v}=\mathbf{v}_{s}+\mathbf{v}_{p}=\nabla \times \phi(x, y) \mathbf{e}_{z}+\nabla \psi(x, y),
$$

with scalar potentials $\phi(x, y)$ and $\psi(x, y)$. It suggests that the aggregate of particles is dominated by the gradient field, with the particles clustering in the minima of the potential $\psi$, similar to compressible Kraichnan flows [5]. When the velocity field is projected onto the solenoidal part $\mathbf{v}_{s}$ the particle distribution remains essentially uniform and there is no clustering (lower panel of Fig. 1).

The dynamics in this initial period is dominated by the exponential contraction in $\mathbf{v}_{p}$. This follows, e.g. from the advection-diffusion equation for a smooth density $\rho$ with diffusivity $D$,

$$
\partial_{t} \rho=-(\nabla \cdot \mathbf{v}) \rho-(\mathbf{v} \cdot \nabla) \rho+D \Delta \rho,
$$

where the divergence patches cause an exponential variation that typically is faster than the variations of the other terms [12]. The natural time scale $\tau=$ $\left\langle(\nabla \cdot \mathbf{v})^{2}\right\rangle^{-1 / 2} \approx 3.5 \tau_{\eta}$ is approximately the lifetime of a divergence patch which comes out to be about $5 \tau_{\eta}$ 
(half width half maximum of the temporal divergence correlation function). As expected, the divergence-free advection contributes little to the clustering. For the discrete particles we measure the density by coarse graining, i.e. dividing the plane into $256 \times 256$ grid cells and count the particles inside the cells. The maximal number $n_{\max } / N$ with total particle number, $N$, increases initially exponentially, as demonstrated in Fig. 2. For $t>\tau$ the exponentially fast formation crosses over to a slower regime. The accumulation of more particles into larger clusters continues: the inset of Fig. 2 2 shows the integrated probability to find cells with no fewer than $N_{0}$ particles, $s\left(t, N_{0}\right)=\sum_{n=N_{0}}^{N} p(t, n)$, for different values of $N_{0}$ with $1 \leq N_{0} \leq N$. The probabilities continue to vary even when the maxima remain essentially constant.

The divergence also determines the particle distribution $p(n)$, as shown in Fig. 3. In Kraichnan type models this distribution comes out to be lognormal for smooth flows [9,5] and delta-like for non-smooth cases [10]. Although we were limited to moderate resolutions and particle numbers, our data in Fig. 3 are closer to an algebraic distribution which is consistent with the exponential distribution of the divergence (see Fig. 国). Neglecting the diffusion term in (2), the density increases exponentially in the Lagrangian frame, $\gamma(t)=\rho(t) / \rho(0)=$ $\exp \left(-\int_{0}^{t}(\nabla \cdot \mathbf{v}) d t^{\prime}\right)$. For $t \leq 1$ we can simplify the exponent to $\lambda t$ where $\lambda=\nabla \cdot \mathbf{v}$ (in units of $\tau^{-1}$ ). If we assume that the density variations are faster than the changes in the velocity field, we have local fluctuations in the divergence that give rise to locally varying density fluctuations. If $P(\lambda)$ is the probability density function (PDF) for the divergence, then the PDF for $\gamma$ becomes

$$
\tilde{P}(\gamma)=\int d \lambda \delta\left(\gamma-\mathrm{e}^{\lambda t}\right) P(\lambda)=\frac{1}{\gamma t} P(\ln \gamma / t) .
$$

Hence, if the divergence fluctuations are Gaussian, as in smooth Kraichnan flows [9], the PDF of the density fluctuations is lognormal. However, in the case of the surface flow the divergence fluctuations have a filamentary smallscale structure [12] (refered to as a shocklet (negative divergence) in compressible supersonic turbulence [16]). The small scale structures appear in the PDF as expo-

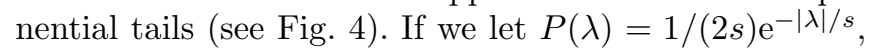
where $s \approx 0.95$ from Fig. 4 , then

$$
P(\gamma) \sim|\gamma|^{-1-1 /(s t)} .
$$

As shown in the inset of Fig. 3, the slope $-\alpha$ in the tails of the distribution decreases with time, from about -3.0 at $t=0.5$ to -2.3 at $t=1$, in good agreement with the prediction from (画. For much longer times the discretness of the particles shows up and the distribution ceases to change, for $t=21$ we got $\alpha=1.5$.

The change in behavior for times larger than about 3 (in units of the divergence time) is connected with the discretness of the particles. The exponential contraction near the minima in the potential leads to a depletion of particles in the neighborhood, so that the density cannot increase further by accumulation once all particles that initially were in a region with negative divergence are collected in a cell. Estimates of the size of the cells can be based on the spatial correlations of the divergence field, $C\left(r_{i}\right)=\left\langle\nabla \cdot \mathbf{v}\left(r_{i 0}+r_{i}\right) \nabla \cdot \mathbf{v}\left(r_{i 0}\right)\right\rangle$ with $r_{i}=x, y$. The typical extension of the patches, identified from the first zero of the correlations, is about $20 \eta$ (inset of Fig. (1). Based on this decorrelation length $l_{d} \approx 20 \eta$ the typical maximal number of particles is about $n / N \sim l_{d}^{2} / L^{2} \sim 0.01$, in good agreement with the data in Fig. 2.

As a second set of characteristics we consider the single-particle dispersion, $\sigma(t)$, and the two-particle pair (or relative) dispersion, $d(t)$. The first one is defined as the root mean square of the absolute particle displacement, $\sigma(t)=\left\langle\left[\mathbf{x}\left(t ; \mathbf{x}_{0}, 0\right)-\mathbf{x}_{0}\right]^{2}\right\rangle_{\mathcal{L}}^{1 / 2}$, where $\langle\cdot\rangle_{\mathcal{L}}$ denotes an average over the single Lagrangian particles. The second uses the difference $\mathbf{R}_{12}(t)=\mathbf{x}_{1}\left(t ; \mathbf{x}_{1,0}, 0\right)-\mathbf{x}_{2}\left(t ; \mathbf{x}_{2,0}, 0\right)$ between Lagrangian particle tracks that start at $\mathbf{x}_{1,0}$ and $\mathbf{x}_{2,0}$ and is defined as the root mean square value for all particles pairs, $d(t)=\left\langle\left[\mathbf{R}_{12}(t)-\mathbf{R}_{12}(0)\right]^{2}\right\rangle_{\mathcal{L}}^{1 / 2}$. In order to fix the dependence on initial separation we take it to be about $1 \eta$, with random orientation in space. The singleparticle dispersion reflects the influence of flow structures at different scales on the particle motion and the relative dispersion can detect the clustering, an interparticle property. In two-dimensional incompressible flows, the limiting cases for both quantities are well-known [17. Both quantities have a ballistic regime, $\sim t$, for short times when particle distances lie within the viscous subrange. For times much larger than the Lagrangian integral time scale, $T_{L}$, correlations can be expected to have decayed, and the relative or single particle motion becomes statistically independent and both dispersions increase diffusively as in an uncorrelated Brownian motion, i.e. $\sim t^{1 / 2}$. For intermediate times, anomalous scaling, $\sim t^{\beta}$ with $1 / 2<\beta<1$ has been observed. For an inverse Kolmogorov cascade, pair dispersion scaling exponents were found to be close to the classical Richardson value of $3 / 2$ [18 in numerical simulations 19,20] as well as experiments [21], but, e.g., sensitive to initial pair separation. In the case of single particle dispersion transient trapping of tracers in coherent vortex structures [17] affects the value of $\beta$.

Results on $\sigma(t)$ and $d(t)$ for the full surface flow and

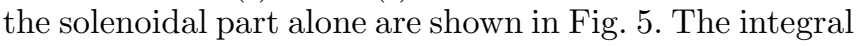
length scales are $T_{L} / \tau_{\eta}=9.1$ for $\mathbf{v}$ and slightly shorter, $T_{L} / \tau_{\eta}=7.8$, for $\mathbf{v}_{s}$. In all cases we do observe the initial ballistic regime up to $T_{L}$. The single-particle dispersion crosses over to the Brownian regime, $\sigma(t) \sim t^{1 / 2}$, for $t>T_{L}$ in both flow fields as indicated in the upper panel of Fig. . We connect this behavior to the fastly varying divergence patches 12 that cause a kind of stochastic sweeping of the tracers.

For intermediate times pairs separate superdiffusively like $d(t) \sim t^{\beta}$ with an exponent of about 1.6, a value that is close to the Richardson prediction, $d_{R}(t) \sim t^{3 / 2}$. Small 
differences may also be attributed to the additional fact that the surface flow was found to have larger intermittency corrections than the associated three-dimensional volume turbulence [11]. Similar scaling behavior is observed for the pair advection in the solenoidal part only (see the lower panel of Fig. 5 for both).

While this anomalous scaling continues for advection in $\mathbf{v}_{s}$ to even larger times, we find a change of the pair dispersion to an almost ballistic behavior of $d(t) \sim t^{0.9}$ for $t \gtrsim 50 \tau_{\eta}$, that does not seem to cross over into a Brownian regime. Microscopically, this means that while one particle follows its partner within a pair, pair correlations decay more slowly and $d(t)$ grows more rapidly than in the Brownian case. Such almost ballistic scaling was also found for the single-particle dispersion in the strongly compressible one-dimensional KuramotoSivashinsky equation [22]. The difference to our case might be caused due to the dimensionality of the problem and the specific character of our surface flow. Superimposed on this process is a chaotic component that comes from $\mathbf{v}_{s}$ and causes exponential separation. Another separation mechanism is a breaking of larger particle clusters due the rapidly emerging flow, i.e. due to the rapidly changing divergence patterns. Clustering and separation were found to be competing processes that cause anomalous diffusion on a longer transient phase of the evolution.

The exponential concentration described above has implications for the dynamics of (inertial) particles that are not density matched with the fluid in which they move. The relations by Maxey and Riley 23 for their motion implies that the velocity field of the particles is not divergence free. The particles will then cluster exponentially, as in Eq. (3). With a view towards the formation of rain [6] there is a uniform condensation of droplets from thermodynamic nucleation and then an exponential clustering to form larger drops, which then fall to the ground as rain drops. The sizes of clusters thus range from the small scale droplets to the size of rain drops and their size distribution thus reflects the distribution of divergence fluctuations by Eq. (3).

We thank J. R. Cressman, J. Davoudi, W. I. Goldburg, E. Hascoët, V. Horvath, K. R. Sreenivasan, and P. K. Yeung for discussions, and the John von Neumann-Institut für Computing in Jülich for support and computing time on a Cray T90. One of us (J.S.) would like to thank the Alexander von Humboldt Foundation for support within the Feodor-Lynen program and Yale University for support and hospitality. This work was supported in part by the European Community, HPRN-CT-2000-00162.

[1] S. Ott and J. Mann, J. Fluid Mech. 422, 207 (2000).

[2] A. La Porta, G. A. Voth, A. M. Crawford, J. Alexander, and E. Bodenschatz, Nature (London) 409, 1017 (2001).
[3] N. Mordant, P. Metz, O. Michel, and J.-F. Pinton, Phys. Rev. Lett. 87, 214501 (2001).

[4] A. Pumir, B. I. Shraiman, and M. Chertkov, Phys. Rev. Lett. 85, 5324 (2000); A. Celani and M. Vergassola, Phys. Rev. Lett. 86, 424 (2001).

[5] G. Falkovich, K. Gawȩdzki, and M. Vergassola, Rev. Mod. Phys. 73, 913 (2001).

[6] E. Balkovsky, G. Falkovich, and A. Fouxon, Phys. Rev. Lett. 86, 2790 (2001).

[7] B. U. Felderhof and G. Ooms, Eur. J. Mech. B 9, 349 (1990); B. van Haarlem, B. J. Boersma, and F. T. M. Nieuwstadt, Phys. Fluids 10, 2608 (1998).

[8] T. M. Antonsen, A. Namenson, E. Ott, and J. C. Sommerer, Phys. Rev. Lett. 75, 3438 (1995).

[9] V. I. Klyatskin and A. I. Saichev, JETP 84, 716 (1997).

[10] K. Gawȩdzki and M. Vergassola, Physica D 183, 63 (2000).

[11] W. I. Goldburg, J. R. Cressman, Z. Vörös, B. Eckhardt, and J. Schumacher, Phys. Rev. E 63, 065303(R) (2001).

[12] B. Eckhardt and J. Schumacher, Phys. Rev. E 64, 016314 (2001).

[13] P. K. Yeung and S. B. Pope, J. Comput. Phys. 79, 373 (1988).

[14] A. L. Rovelstad, R. A. Handler, and P. S. Bernard, J. Comput. Phys. 110, 190 (1994).

[15] J. Eggers and S. Grossmann, Phys. Fluids A 3, 1985 (1991).

[16] D. H. Porter, P. R. Woodward, and A. Pouquet, Phys. Fluids 10, 237 (1998).

[17] D. Elhmaidi, A. Provenzale, and A. Babiano, J. Fluid Mech. 257, 533 (1993).

[18] L. F. Richardson, Proc. Roy. Soc. A 110, 709 (1926).

[19] N. Zouari and A. Babiano, Physica D 76, 318 (1994).

[20] G. Boffetta and A. Celani, Physica A 280, 1 (2000).

[21] M. C. Jullien, J. Paret, and P. Tabeling, Phys. Rev. Lett. 82, 2872 (1999).

[22] T. Bohr and A. Pikovsky, Phys. Rev. Lett. 70, 2892 (1993).

[23] M. Maxey and J. Riley, Phys. Fluids 26, 883 (1983). 

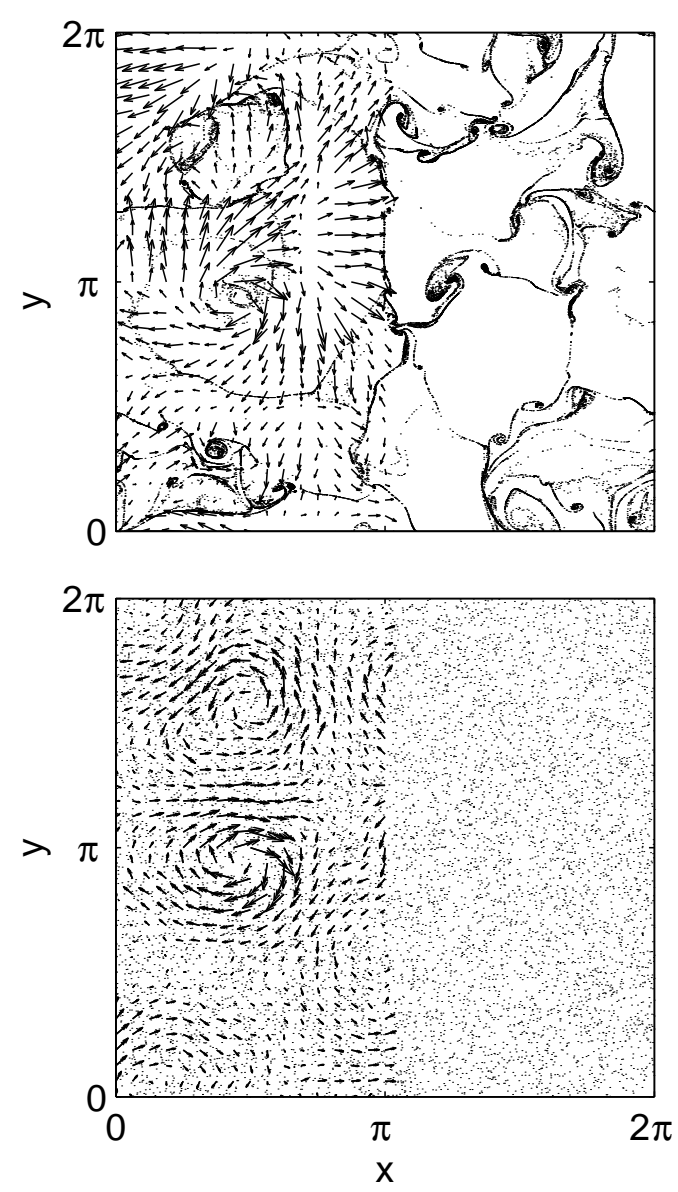

FIG. 1. Distribution of 36000 tracers and the instantaneous velocity field. Upper panel: full flow v. Lower panel: solenoidal flow $\mathbf{v}_{s}$. The snapshots for both cases were taken at $t / \tau_{\eta}=21$ after the start. In order to highlight the tracer patterns the underlying flow fields are shown in one half of the box.

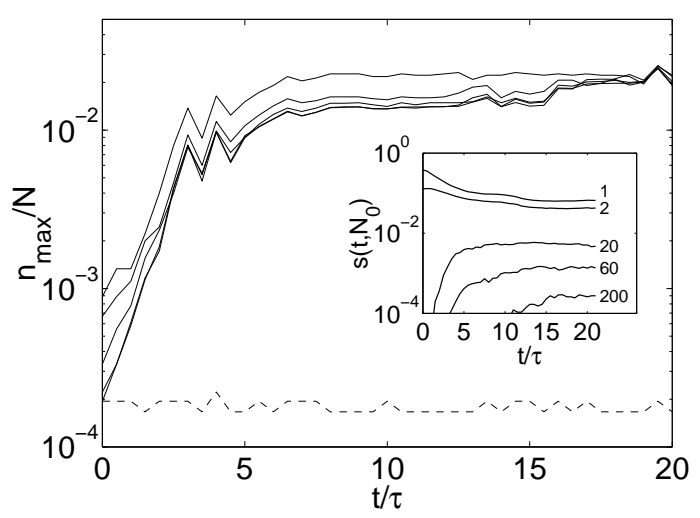

FIG. 2. Maximum particle number per cell $n_{\max } / N$ vs. $t / \tau$ for particle numbers 36000, 18000, 9000, 4500, and 2250 (from bottom to top). Dashed line is for $\mathbf{v}_{\mathbf{s}}$ and solid lines are for $\mathbf{v}$. The inset shows the temporal evolution of the integrated probability $s\left(t, N_{0}\right)$ with values of $N_{0}$ to the right.

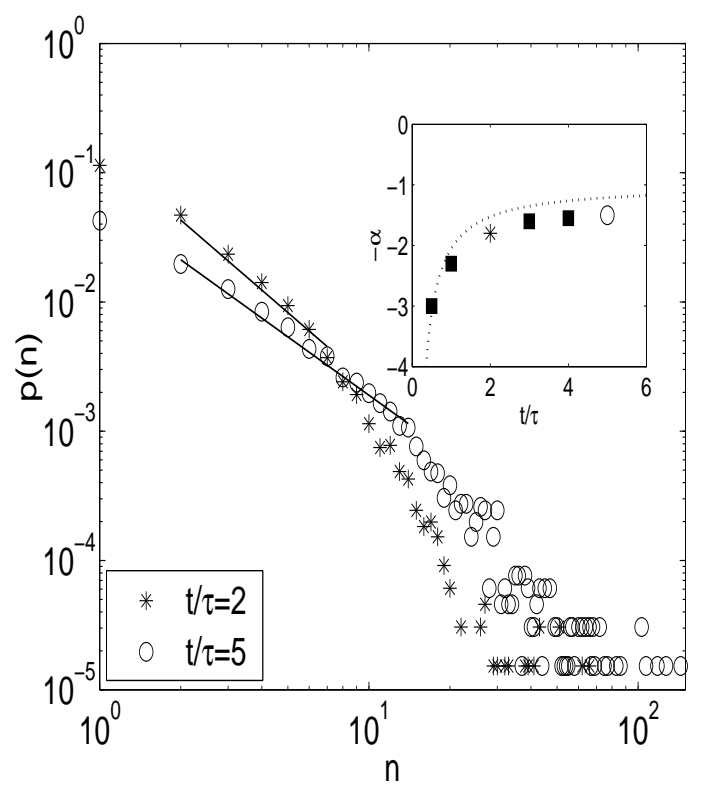

FIG. 3. Probability density $p(n)$ for different times during the cluster evolution. Straight lines indicate fits with an algebraic law. The inset shows the exponents $\alpha$ of $p(n) \sim n^{-\alpha}$ as a function of time $t / \tau$. The dotted line follows from (4).

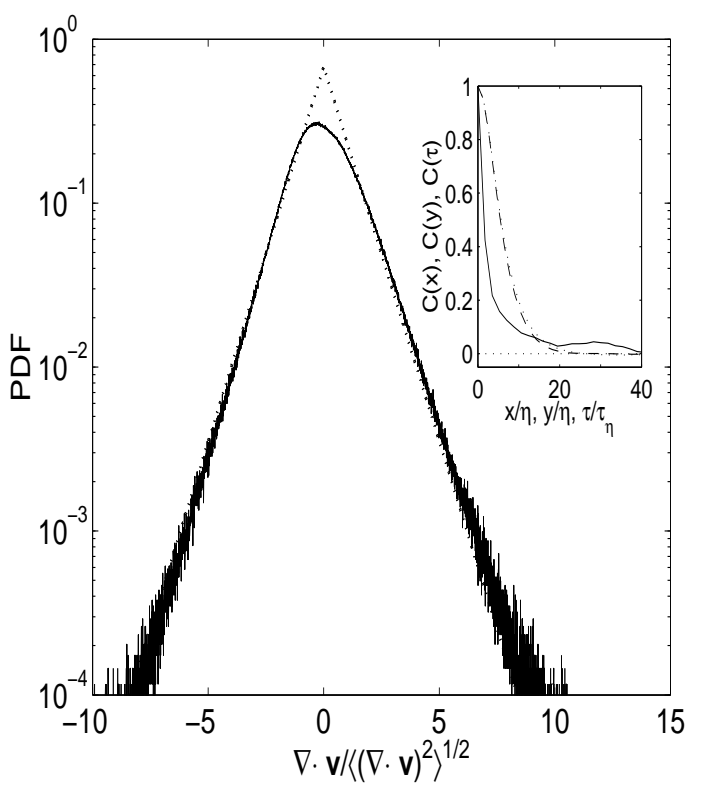

FIG. 4. PDF of the divergence of the surface flow $\mathbf{v}$. The dotted line is an exponential fit, $p(\lambda)=0.68 \exp (-|\lambda| / 0.95)$ with $\lambda=(\nabla \cdot \mathbf{v}) \tau$. The inset shows the spatial and temporal correlation functions of the divergence field, $C(x)$ over $x / \eta$, $C(y)$ over $y / \eta$, and $C(\tau)$ over $\tau / \tau_{\eta}$. 


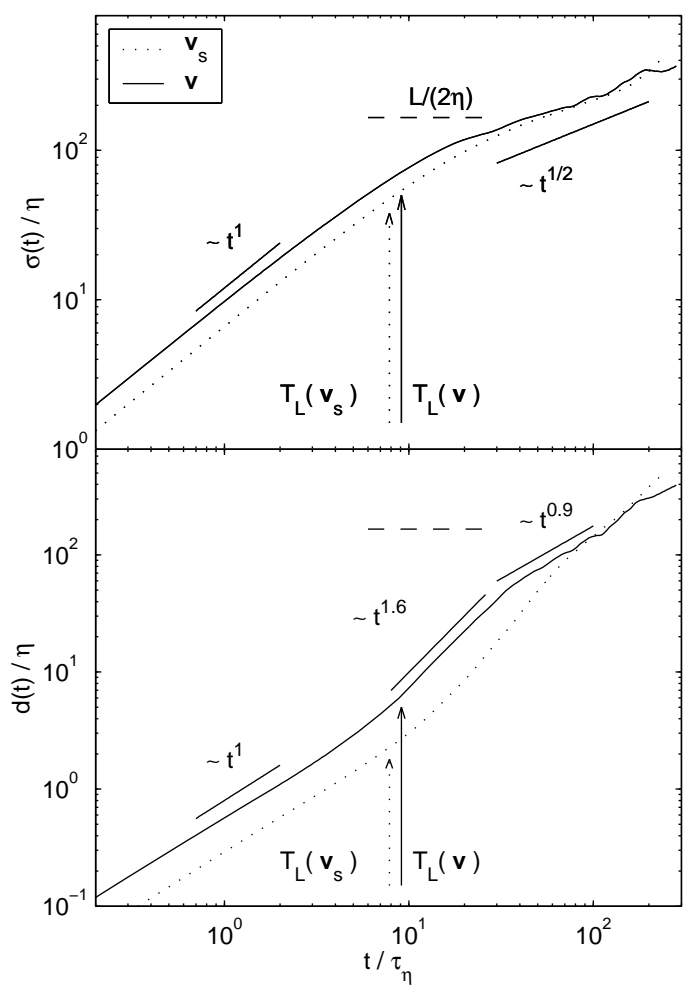

FIG. 5. Upper panel: Single-particle dispersion, $\sigma(t)$, as a function of time. Solid curve is for advection by the full surface flow $\mathbf{v}$, while the dotted one is for advection by the solenoidal part $\mathbf{v}_{s}$ only. The Lagrangian integral time scales for both fields are indicated by arrows. Lower panel: Two-particle pair dispersion, $d(t)$, as a function of time. Linestyles are as above. The dashed horizontal lines indicate half the box size. 